\title{
Automation of the process of reverse engineering of urban development projects based on the results of aerial photographic operations
}

\author{
Elena A kulova ${ }^{1}$, Aleksandr Alybaev² , and Daria Buzina ${ }^{1}$ \\ ${ }^{1}$ U ral State M ining U niversity, 620144 Ekaterinburg, Russia \\ ${ }^{2}$ U ral-Siberian Geospatial Company, 620144 Ekaterinburg, R ussia
}

\begin{abstract}
D models of objects of urban development, created by the results of aerial photographic operations or laser scanning, have high metric accuracy, but require significant labor costs, both in terms of creation and visualization. To construct three-dimensional models that allow the user to perceive information in the usual spatial form, and satisfying the accuracy requirements for solving urban planning problems, it is proposed to automate the reverse engineering process based on the $M$ etashape and ContextC apture software products.
\end{abstract}

Currently, Russia is implementing the national program "Digital Economy of the Russian Federation", in which much attention is paid to the development of geographic information technologies and the use of spatial data. The Smart City section, included in this program, is aimed at creating an effective urban management system, creating safe and comfortable living conditions for people. The development of this section involves the use of spatial data to construct digital models of buildings and structures, as well as for further operational management of urban areas [1].

Spatial data - digital data about spatial objects, including information about their shape, location and properties, including those presented with the use of coordinates. For the digital economy, spatial data is required in the form of three-dimensional coordinates $\mathrm{X}, \mathrm{Y}, \mathrm{Z}$ as well as information about the reliability and accuracy of their determination.

The digital three-dimensional model of geospace is a system of a number of elements: a three-dimensional model of the earth's surface, ground objects (real estate objects) and subsoil (geological models), etc. [4]. Spatial models are the basis of geoinformational design. 3D model can be created either manually (using CAD or GIS) or automatically. The initial data for building three-dimensional models are, as a rule, the data of geodetic and photogrammetric definitions. Digital models built according to various technological schemes have a number of advantages and disadvantages (table 1). 
Table 1. Advantages and disadvantages of the main various types of three-dimensional models.

\begin{tabular}{|c|c|c|c|c|c|}
\hline \multicolumn{6}{|c|}{ THREE-DIMENSIONAL MODEL TYPE } \\
\hline \multicolumn{2}{|c|}{ 3D vector model } & \multicolumn{2}{|c|}{$\begin{array}{c}\text { 3D model, created } \\
\text { automatically }\end{array}$} & \multicolumn{2}{|c|}{ 3D stereomodel } \\
\hline+ & - & + & - & + & - \\
\hline $\begin{array}{c}\text { high } \\
\text { geometric } \\
\text { detailing }\end{array}$ & $\begin{array}{l}\text { complexity } \\
\text { of creation }\end{array}$ & $\begin{array}{l}\text { high speed of } \\
\text { creation }\end{array}$ & $\begin{array}{l}\text { Low geometric } \\
\text { accuracy }\end{array}$ & $\begin{array}{l}\text { high speed } \\
\text { of creation }\end{array}$ & $\begin{array}{c}\text { can be viewed } \\
\text { only using a } \\
\text { stereo }\end{array}$ \\
\hline \multirow[t]{2}{*}{$\begin{array}{l}\text { textures } \\
\text { do not } \\
\text { contain } \\
\text { images of } \\
\text { foreign } \\
\text { objects }\end{array}$} & $\begin{array}{l}\text { High metric } \\
\text { accuracy }\end{array}$ & $\begin{array}{c}\text { High } \\
\text { photorealism }\end{array}$ & $\begin{array}{l}\text { impossibility to } \\
\text { separate building } \\
\text { objects from the } \\
\text { surface or from } \\
\text { each other }\end{array}$ & \multirow[t]{2}{*}{$\begin{array}{l}\text { High metric } \\
\text { accuracy; } \\
\text { most } \\
\text { capacious of } \\
\text { all types }\end{array}$} & $\begin{array}{l}\text { monitor and } \\
\text { stereo glasses }\end{array}$ \\
\hline & Iow realism & $\begin{array}{l}\text { low cost of } \\
\text { model } \\
\text { creation }\end{array}$ & $\begin{array}{l}\text { Iow quality } \\
\text { textures }\end{array}$ & & \\
\hline
\end{tabular}

Spatial body modeling can create functionally identical objects. The differences between them are mainly differences in how they are created and edited, and agreements on use in different areas, as well as differences in the types of approximations between the model and the reality [9].

Visualization of the urban environment model is widely used to solve various problems in such fields of activity as construction, urban management, education, culture, etc. Threedimensional visualization allows the user to perceive information in a familiar spatial form [7].

Building a 3D model from scratch is labor or time consuming. For such cases, the software offers the best option: creating a mathematical 3D model or surface (set of surfaces) based on information obtained from a physical object. Currently, the term "reverse engineering" or "reverse design" for the most part refers to the process of obtaining a digital 3D model of a real product using automated design systems. The process begins with a 3D scanning, in which the shape of the object is converted into a mathematical image in the form of a point cloud. For this purpose, laser scanners, structured white or blue light devices, coordinate measuring machines (CMMs) and computed tomography are used. Reverse engineering is the process of designing a digital model that describes an object and its technological properties by performing a comprehensive analysis of its structure. This process is aimed at creating a virtual 3D model based on an existing physical object for its study, duplication or improvement [5].

In this paper, it is proposed to use aerial photographic data and application software packages that allow the implementation of this process to be used as starting materials for the implementation of reverse engineering technology.

Automatic designing is performed in special software products that contain algorithms for restoring the geometric shape of objects and building textures of building facades from their stereo images. The technological process of building a three-dimensional model involves the identification of characteristic points in the zone of mutual overlap of images, the formation of a point cloud that describes the earth's surface and objects towering above it. In the next step, the point cloud is triangulated to get the surface. In the resulting surface, a search for planes is performed for the best transfer of walls and roofs of buildings. The final product is a terrain three-dimensional model, presented with varying degrees of detailing.

Currently, 3D stereo models are created using the reverse engineering method based on aerial photography results using the PHOTOMOD software product. However, for this purpose, there are a number of software products on the Russian market that automate the 
process of preparing a digital model and significantly reduce the time of desk processing. These software products include Metashape and ContextCapture.

In the Metashape and ContextCapture programs, two 3D models of the same territory of the city, represented by objects of different geometric structures, were built. When comparing the models, it can be seen that in the ContextCapture program the buildings look quite realistic, and their walls are perpendicular to the surface of the earth (Figure 1, number 2). In the Metashape program, buildings are more rounded, blurred, without distinct right angles (Figure 1, number 1).
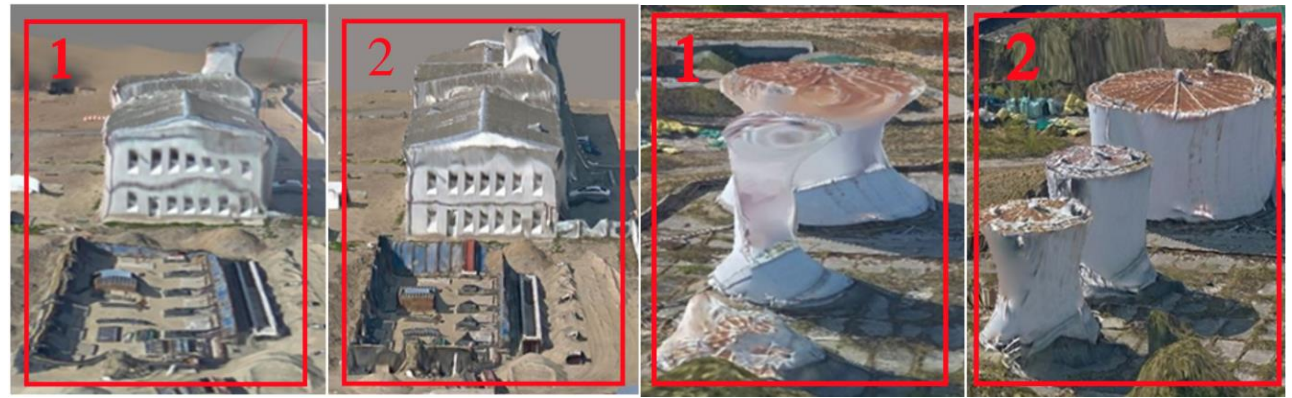

Fig. 1. 3D model of a city site in $M$ etashape and ContextC apture, respectively

In the ContextCapture program, construction of objects can be performed not only in the form of a parallelepiped (Figure 1, number 1). The figure shows that three structures with a cylindrical shape are pointed almost without distortion. In the Metashape program, one of the cylinders was not built at all, and the other two do not have a clear outline (Figure 1, number 2).

The walls of buildings under construction in the ContextCapture program (Figure 2, number 2) and in the Metashape program (Figure 3, number 1), are completely blended with the ground.
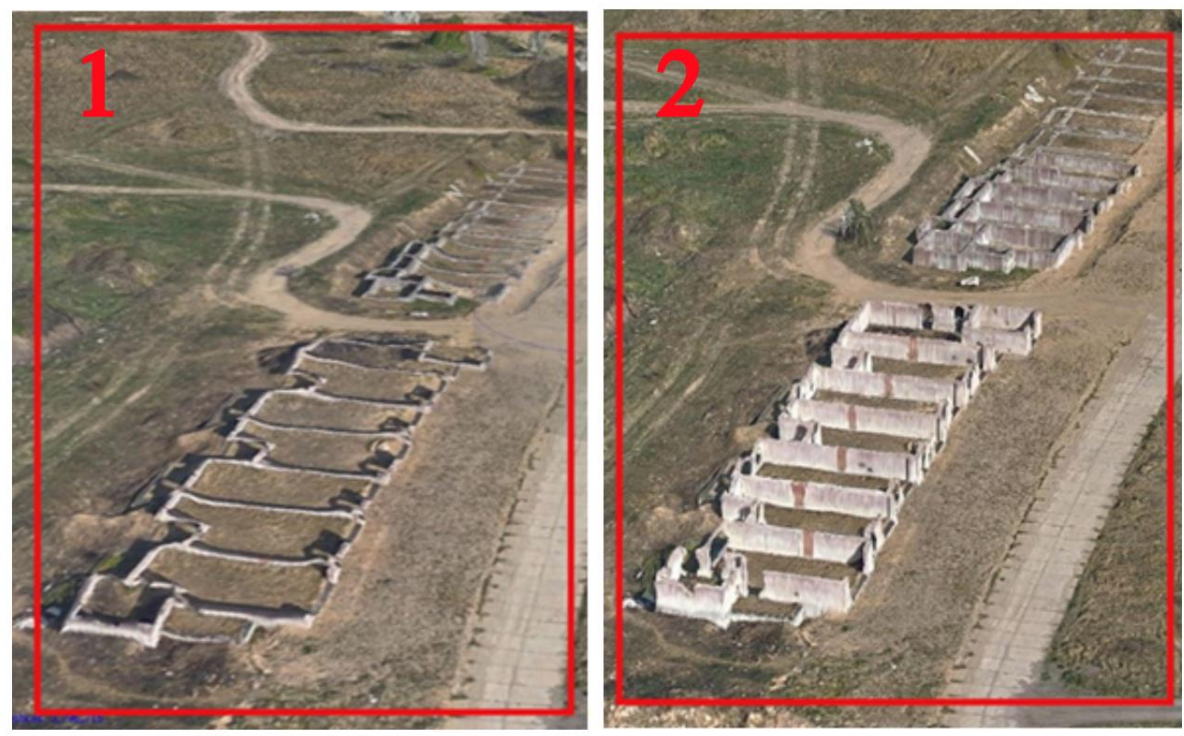

Fig. 2. 3D model of a city site in M etashape and ContextC apture, respectively. 
During a visual comparison of models built in the ContextCapture and Metashape programs, the advantages and disadvantages of each software product were identified (table 2).

Table 2. Formatting sections, subsections and subsubsections.

\begin{tabular}{|c|c|c|c|c|}
\hline \multirow[t]{2}{*}{ CRITERION } & \multicolumn{2}{|c|}{ METASHAPE } & \multicolumn{2}{|c|}{ CONTEXTCAPTURE } \\
\hline & + & - & + & - \\
\hline 3D model texture & $\begin{array}{l}\text { Clear texture } \\
\text { prevails }\end{array}$ & $\begin{array}{l}\text { Grainy texture is } \\
\text { found }\end{array}$ & $\begin{array}{l}\text { Clear texture, no } \\
\text { distortion }\end{array}$ & \\
\hline 3D model realism & In thes & wo programs, a rea & ic 3D model of the & ritory is built. \\
\hline $\begin{array}{c}\text { Buildings } \\
\text { construction }\end{array}$ & $\begin{array}{c}\text { Buildings } \\
\text { construction in } \\
\text { progress }\end{array}$ & $\begin{array}{c}\text { M ost buildings } \\
\text { have irregular } \\
\text { geometric shapes. }\end{array}$ & $\begin{array}{c}\text { Pretty clear } \\
\text { geometric shape } \\
\text { respected }\end{array}$ & $\begin{array}{l}\text { In single cases, an } \\
\text { irregular geometric } \\
\text { shape is found }\end{array}$ \\
\hline $\begin{array}{c}\text { Drawing of facades, } \\
\text { windows and roofs } \\
\text { of buildings }\end{array}$ & $\begin{array}{c}\text { Elements are } \\
\text { displayed }\end{array}$ & $\begin{array}{l}\text { M ost often, the } \\
\text { elements are } \\
\text { blurred. }\end{array}$ & $\begin{array}{l}\text { Clear drawing of } \\
\text { elements }\end{array}$ & $\begin{array}{c}\text { Blurred elements are } \\
\text { rare. }\end{array}$ \\
\hline $\begin{array}{c}\text { Construction of } \\
\text { fences and low } \\
\text { elements }\end{array}$ & & $\begin{array}{l}\text { Completely blend } \\
\text { with the surface }\end{array}$ & $\begin{array}{l}\text { Do not blend } \\
\text { with the surface }\end{array}$ & \\
\hline $\begin{array}{c}\text { Program } \\
\text { availability }\end{array}$ & $\begin{array}{l}\text { Widespread in } \\
\text { Russia }\end{array}$ & & & Rarely used in Russia \\
\hline
\end{tabular}

After analyzing the advantages and disadvantages of each software product, it was concluded that the further construction of $3 \mathrm{D}$ models will be performed in the ContextCapture program.

To determine the possibilities of using this program to create three-dimensional models of cultural heritage objects, a 3D model of a cultural heritage object of regional significance "Outhouse from the Plotnikovs Manor" was built in the city of Tobolsk (Figure 3-4).

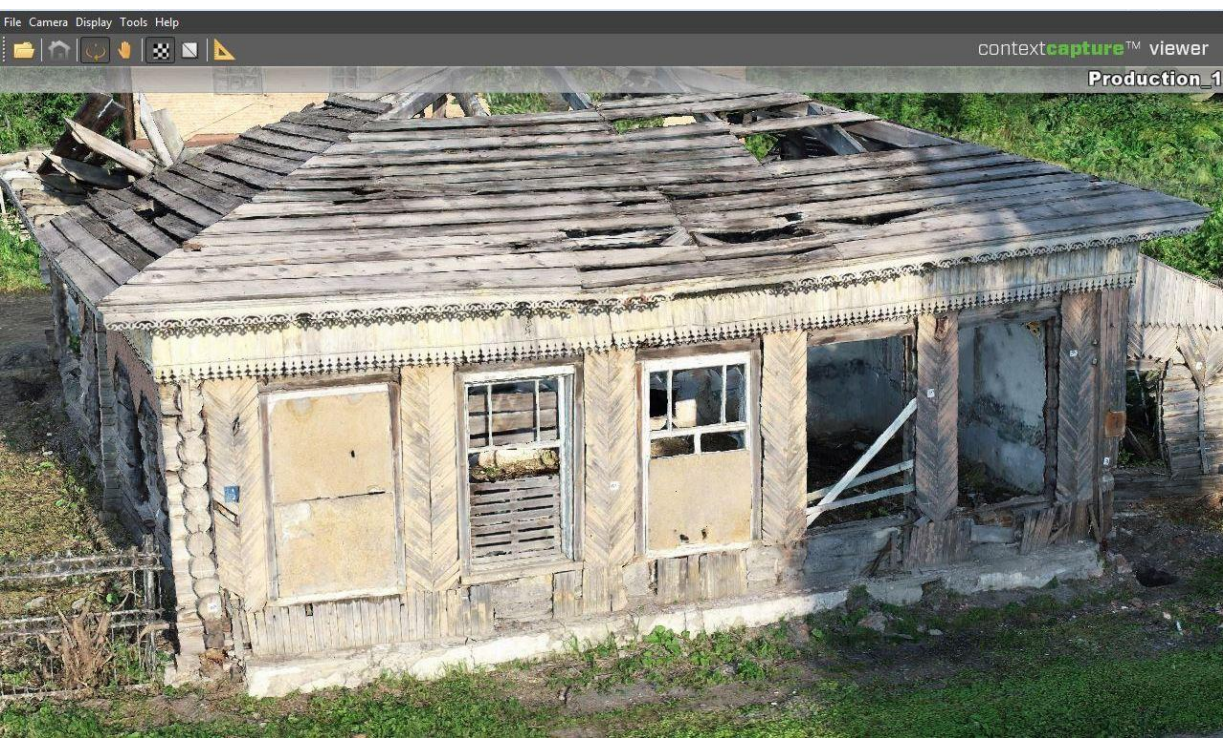

Fig. 3. 3D model of the cultural heritage object of regional significance "Outhouse from the Plotnikovs Manor" in the ContextCapture program. 


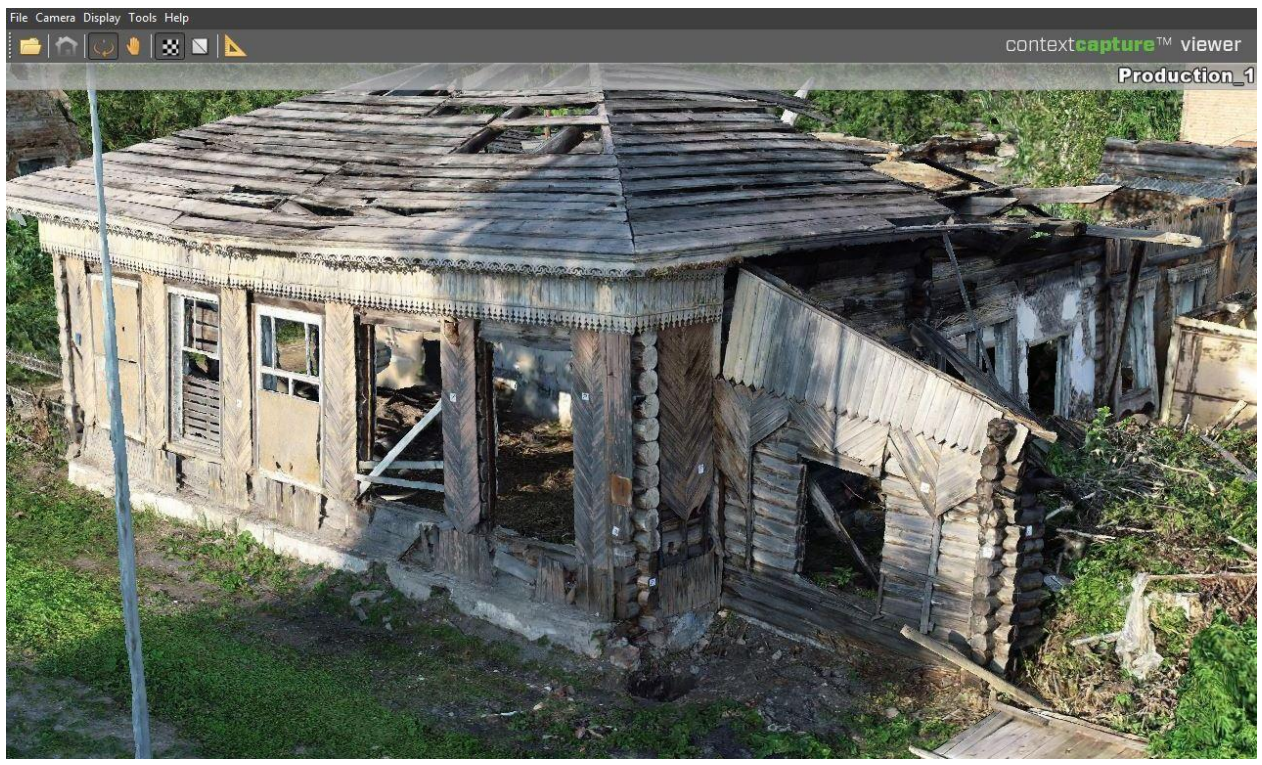

Fig. 4. 3D model of the cultural heritage object of regional significance "Outhouse from the Plotnikovs Manor" in the ContextCapture program.

To automatically create a 3D model, aerial photographs obtained by performing several types of aerial photogrammetric survey (hereinafter - APS) were used:

- planned APS from a height of $30 \mathrm{~m}$ from the building. Overlap $80 / 80 \%$;

- planned APS from a height of $15 \mathrm{~m}$ from the building. Overlap 80/80\%;

- perspective (oblique) APS around the building from a height of $30 \mathrm{~m}$ from the building, a distance of $30 \mathrm{~m}$ from the corners of the building;

- perspective (oblique) APS around the building from a height of $15 \mathrm{~m}$ from the building, a distance of $15 \mathrm{~m}$ from the corners of the building;

- perspective (oblique) APS around the building from a height of $2 \mathrm{~m}$ from the ground, a distance of $15 \mathrm{~m}$ from the corners of the building;

Aerial photography was carried out using the DJI PHANTOM 4 PRO quadcopter, and markers were also placed on the walls of the building.

The three-dimensional model of a cultural heritage object of regional significance was created to compare the three-dimensional coordinates (X, Y and Z) of characteristic points on a 3D model obtained automatically and on a 3D stereo model built in PHOTOMOD. Sixteen wall markers and eleven characteristic points were selected for measurement, some of which are marked in Figure 6.
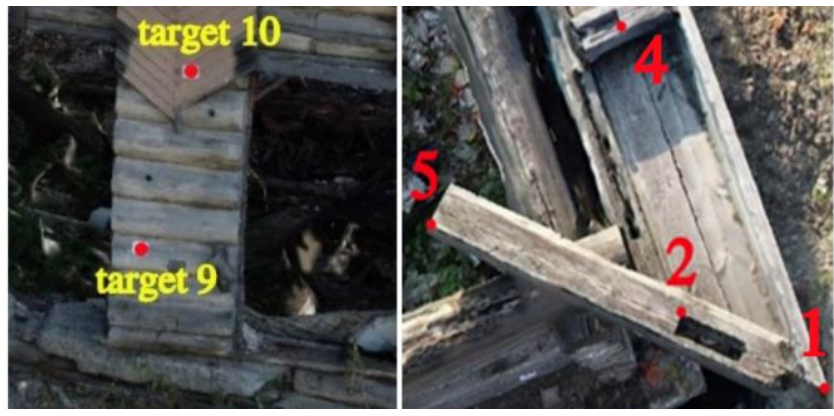

Fig. 4. Fragment of a 3D model with several wall markers and characteristic points involved in measurements. 
Table 3 presents the coordinates of wall markers and characteristic points, as well as the measurement tolerances $\Delta \mathrm{X}, \Delta \mathrm{Y}, \Delta \mathrm{S}, \Delta \mathrm{Z}$.

Table 3. The coordinates of sixteen wall markers and eleven characteristic points with measurement tolerances $\Delta \mathrm{X}, \Delta \mathrm{Y}, \Delta \mathrm{S}, \Delta \mathrm{Z}$.

\begin{tabular}{|c|c|c|c|c|c|c|c|c|c|}
\hline \multicolumn{3}{|c|}{ 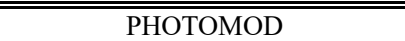 } & \multicolumn{3}{|c|}{ 3D MODEL } & \multicolumn{4}{|c|}{ T TOLERANCE } \\
\hline \multicolumn{10}{|c|}{ target 28} \\
\hline $\mathrm{X}, \mathrm{m}$ & $\mathrm{Y}, \mathrm{m}$ & $\mathrm{Z}, \mathrm{m}$ & $\mathrm{X}, \mathrm{m}$ & $\mathrm{Y}, \mathrm{m}$ & $\mathrm{Z,m}$ & $\Delta \mathrm{X}, \mathrm{m}$ & $\Delta \mathrm{Y}, \mathrm{m}$ & $\Delta \mathrm{S}, \mathrm{m}$ & $\Delta \mathrm{Z}, \mathrm{m}$ \\
\hline 101.3057 & 105.5167 & 102.7466 & 101.3050 & 105.5190 & 102.7480 & 0.001 & -0.002 & 0.002 & -0.001 \\
\hline \multicolumn{10}{|c|}{ target 22} \\
\hline $\mathrm{X}, \mathrm{m}$ & $\mathrm{Y}, \mathrm{m}$ & $\mathrm{Z}, \mathrm{m}$ & $\mathrm{X}, \mathrm{m}$ & $\mathrm{Y}, \mathrm{m}$ & $\mathrm{Z}, \mathrm{m}$ & $\Delta \mathrm{X}, \mathrm{m}$ & $\Delta \mathrm{Y}, \mathrm{m}$ & $\Delta \mathrm{S}, \mathrm{m}$ & $\Delta \mathrm{Z}, \mathrm{m}$ \\
\hline 108.4479 & 108.4041 & 102.8614 & 108.4530 & 108.4020 & 102.8650 & -0.005 & 0.002 & 0.005 & -0.004 \\
\hline \multicolumn{10}{|c|}{ target 20} \\
\hline $\mathrm{X}, \mathrm{m}$ & $\mathrm{Y}, \mathrm{m}$ & $\mathrm{Z}, \mathrm{m}$ & $\mathrm{X}, \mathrm{m}$ & $\mathrm{Y}, \mathrm{m}$ & $\mathrm{Z}, \mathrm{m}$ & $\Delta \mathrm{X}, \mathrm{m}$ & $\Delta \mathrm{Y}, \mathrm{m}$ & $\Delta \mathrm{S}, \mathrm{m}$ & $\Delta \mathrm{Z}, \mathrm{m}$ \\
\hline 104.1673 & 111.4815 & 101.5908 & 104.1760 & 111.4980 & 101.5910 & -0.009 & -0.017 & 0.019 & 0.000 \\
\hline \multicolumn{10}{|c|}{ target 19} \\
\hline $\mathrm{X}, \mathrm{m}$ & $\mathrm{Y}, \mathrm{m}$ & $\mathrm{Z}, \mathrm{m}$ & $\mathrm{X}, \mathrm{m}$ & $\mathrm{Y}, \mathrm{m}$ & $\mathrm{Z}, \mathrm{m}$ & $\Delta \mathrm{X}, \mathrm{m}$ & $\Delta \mathrm{Y}, \mathrm{m}$ & $\Delta \mathrm{S}, \mathrm{m}$ & $\Delta \mathrm{Z}, \mathrm{m}$ \\
\hline 105.1736 & 110.8782 & 102.0341 & 105.1880 & 110.8760 & 102.0400 & -0.014 & 0.002 & 0.014 & -0.006 \\
\hline \multicolumn{10}{|c|}{ target 16} \\
\hline $\mathrm{X}, \mathrm{m}$ & $\mathrm{Y}, \mathrm{m}$ & $\mathrm{Z}, \mathrm{m}$ & $\mathrm{X}, \mathrm{m}$ & $\mathrm{Y}, \mathrm{m}$ & $\mathrm{Z}, \mathrm{m}$ & $\Delta \mathrm{X}, \mathrm{m}$ & $\Delta \mathrm{Y}, \mathrm{m}$ & $\Delta \mathrm{S}, \mathrm{m}$ & $\Delta \mathrm{Z}, \mathrm{m}$ \\
\hline 107.8736 & 109.1401 & 100.6807 & 107.8800 & 109.1610 & 100.6900 & -0.006 & -0.021 & 0.022 & -0.009 \\
\hline \multicolumn{10}{|c|}{ target 15} \\
\hline $\mathrm{X}, \mathrm{m}$ & $\mathrm{Y}, \mathrm{m}$ & $\mathrm{Z}, \mathrm{m}$ & $\mathrm{X}, \mathrm{m}$ & $\mathrm{Y}, \mathrm{m}$ & $\mathrm{Z}, \mathrm{m}$ & $\Delta \mathrm{X}, \mathrm{m}$ & $\Delta \mathrm{Y}, \mathrm{m}$ & $\Delta \mathrm{S}, \mathrm{m}$ & $\Delta \mathrm{Z}, \mathrm{m}$ \\
\hline 108.2822 & 108.9947 & 101.3130 & 108.2900 & 108.9940 & 101.3210 & -0.008 & 0.001 & 0.008 & -0.008 \\
\hline \multicolumn{10}{|c|}{ target 13} \\
\hline $\mathrm{X}, \mathrm{m}$ & $\mathrm{Y}, \mathrm{m}$ & $\mathrm{Z}, \mathrm{m}$ & $\mathrm{X}, \mathrm{m}$ & $\mathrm{Y}, \mathrm{m}$ & $\mathrm{Z}, \mathrm{m}$ & $\Delta \mathrm{X}, \mathrm{m}$ & $\Delta \mathrm{Y}, \mathrm{m}$ & $\Delta \mathrm{S}, \mathrm{m}$ & $\Delta \mathrm{Z}, \mathrm{m}$ \\
\hline 113.8538 & 98.1493 & 100.8391 & 113.8590 & 98.1553 & 100.8430 & -0.005 & -0.006 & 0.008 & -0.004 \\
\hline \multicolumn{10}{|c|}{ target 12} \\
\hline $\mathrm{X}, \mathrm{m}$ & $\mathrm{Y}, \mathrm{m}$ & $\mathrm{Z}, \mathrm{m}$ & $\mathrm{X}, \mathrm{m}$ & $\mathrm{Y}, \mathrm{m}$ & $\mathrm{Z}, \mathrm{m}$ & $\Delta \mathrm{X}, \mathrm{m}$ & $\Delta \mathrm{Y}, \mathrm{m}$ & $\Delta \mathrm{S}, \mathrm{m}$ & $\Delta \mathrm{Z}, \mathrm{m}$ \\
\hline 113.7231 & 97.6635 & 102.0343 & 113.7210 & 97.6614 & 102.0360 & 0.002 & 0.002 & 0.003 & -0.002 \\
\hline \multicolumn{10}{|c|}{ target 10} \\
\hline $\mathrm{X}, \mathrm{m}$ & $\mathrm{Y}, \mathrm{m}$ & $\mathrm{Z}, \mathrm{m}$ & $\mathrm{X}, \mathrm{m}$ & $\mathrm{Y}, \mathrm{m}$ & $\mathrm{Z}, \mathrm{m}$ & $\Delta \mathrm{X}, \mathrm{m}$ & $\Delta \mathrm{Y}, \mathrm{m}$ & $\Delta \mathrm{S}, \mathrm{m}$ & $\Delta \mathrm{Z}, \mathrm{m}$ \\
\hline 105.7813 & 93.5523 & 102.6012 & 105.7780 & 93.5529 & 102.6000 & 0.003 & -0.001 & 0.003 & 0.001 \\
\hline \multicolumn{10}{|c|}{ target 9} \\
\hline $\mathrm{X}, \mathrm{m}$ & $\mathrm{Y}, \mathrm{m}$ & $\mathrm{Z}, \mathrm{m}$ & $\mathrm{X}, \mathrm{m}$ & $\mathrm{Y}, \mathrm{m}$ & $\mathrm{Z}, \mathrm{m}$ & $\Delta \mathrm{X}, \mathrm{m}$ & $\Delta \mathrm{Y}, \mathrm{m}$ & $\Delta \mathrm{S}, \mathrm{m}$ & $\Delta \mathrm{Z}, \mathrm{m}$ \\
\hline 105.5834 & 93.3735 & 101.3216 & 105.5810 & 93.3699 & 101.3180 & 0.002 & 0.004 & 0.004 & 0.004 \\
\hline \multicolumn{10}{|c|}{ target 7} \\
\hline $\mathrm{X}, \mathrm{m}$ & $\mathrm{Y}, \mathrm{m}$ & $\mathrm{Z}, \mathrm{m}$ & $\mathrm{X}, \mathrm{m}$ & $\mathrm{Y}, \mathrm{m}$ & $\mathrm{Z}, \mathrm{m}$ & $\Delta \mathrm{X}, \mathrm{m}$ & $\Delta \mathrm{Y}, \mathrm{m}$ & $\Delta \mathrm{S}, \mathrm{m}$ & $\Delta \mathrm{Z}, \mathrm{m}$ \\
\hline 98.7419 & 90.7226 & 101.6727 & 98.7312 & 90.7218 & 101.6720 & 0.011 & 0.001 & 0.011 & 0.001 \\
\hline \multicolumn{10}{|c|}{ target 6} \\
\hline $\mathrm{X}, \mathrm{m}$ & $\mathrm{Y}, \mathrm{m}$ & $\mathrm{Z}, \mathrm{m}$ & $\mathrm{X}, \mathrm{m}$ & $\mathrm{Y}, \mathrm{m}$ & $\mathrm{Z}, \mathrm{m}$ & $\Delta \mathrm{X}, \mathrm{m}$ & $\Delta \mathrm{Y}, \mathrm{m}$ & $\Delta \mathrm{S}, \mathrm{m}$ & $\Delta \mathrm{Z}, \mathrm{m}$ \\
\hline 95.4687 & 91.5969 & 101.8743 & 95.4683 & 91.5837 & 101.8690 & 0.000 & 0.013 & 0.013 & 0.005 \\
\hline \multicolumn{10}{|c|}{ target 1} \\
\hline $\mathrm{X}, \mathrm{m}$ & $\mathrm{Y}, \mathrm{m}$ & $\mathrm{Z}, \mathrm{m}$ & $\mathrm{X}, \mathrm{m}$ & $\mathrm{Y}, \mathrm{m}$ & $\mathrm{Z}, \mathrm{m}$ & $\Delta \mathrm{X}, \mathrm{m}$ & $\Delta \mathrm{Y}, \mathrm{m}$ & $\Delta \mathrm{S}, \mathrm{m}$ & $\Delta \mathrm{Z}, \mathrm{m}$ \\
\hline 89.7496 & 99.2619 & 101.4856 & 89.7483 & 99.2625 & 101.4770 & 0.001 & -0.001 & 0.001 & 0.009 \\
\hline \multicolumn{10}{|c|}{ target 3} \\
\hline $\mathrm{X}, \mathrm{m}$ & $\mathrm{Y}, \mathrm{m}$ & $Z, m$ & $\mathrm{X}, \mathrm{m}$ & $\mathrm{Y}, \mathrm{m}$ & $Z, m$ & $\Delta \mathrm{X}, \mathrm{m}$ & $\Delta \mathrm{Y}, \mathrm{m}$ & $\Delta \mathrm{S}, \mathrm{m}$ & $\Delta \mathrm{Z}, \mathrm{m}$ \\
\hline 91.0840 & 96.9191 & 102.5256 & 91.0842 & 96.9209 & 102.5220 & 0.000 & -0.002 & 0.002 & 0.004 \\
\hline \multicolumn{10}{|c|}{ target 22} \\
\hline $\mathrm{X}, \mathrm{m}$ & $\mathrm{Y}, \mathrm{m}$ & $\mathrm{Z}, \mathrm{m}$ & $\mathrm{X}, \mathrm{m}$ & $\mathrm{Y}, \mathrm{m}$ & $\mathrm{Z}, \mathrm{m}$ & $\Delta \mathrm{X}, \mathrm{m}$ & $\Delta \mathrm{Y}, \mathrm{m}$ & $\Delta \mathrm{S}, \mathrm{m}$ & $\Delta \mathrm{Z}, \mathrm{m}$ \\
\hline 108.4479 & 108.4041 & 102.8614 & 108.4490 & 108.4100 & 102.8680 & -0.001 & -0.006 & 0.006 & -0.007 \\
\hline & & & & target 23 & & & & & \\
\hline $\mathrm{X}, \mathrm{m}$ & $\mathrm{Y}, \mathrm{m}$ & $\mathrm{Z}, \mathrm{m}$ & $\mathrm{X}, \mathrm{m}$ & $\mathrm{Y}, \mathrm{m}$ & $Z, m$ & $\Delta \mathrm{X}, \mathrm{m}$ & $\Delta \mathrm{Y}, \mathrm{m}$ & $\Delta \mathrm{S}, \mathrm{m}$ & $\Delta \mathrm{Z}, \mathrm{m}$ \\
\hline 109.5483 & 106.4476 & 102.5378 & 109.5500 & 106.4500 & 102.5360 & -0.002 & -0.002 & 0.003 & 0.002 \\
\hline & & & & No. 1 & & & & & \\
\hline $\mathrm{X}, \mathrm{m}$ & $\mathrm{Y}, \mathrm{m}$ & $\mathrm{Z}, \mathrm{m}$ & $\mathrm{X}, \mathrm{m}$ & $\mathrm{Y}, \mathrm{m}$ & $\mathrm{Z}, \mathrm{m}$ & $\Delta \mathrm{X}, \mathrm{m}$ & $\Delta \mathrm{Y}, \mathrm{m}$ & $\Delta \mathrm{S}, \mathrm{m}$ & $\Delta \mathrm{Z}, \mathrm{m}$ \\
\hline 98.7790 & 88.9700 & 104.3960 & 98.7846 & 88.9823 & 104.4070 & -0.006 & -0.012 & 0.013 & -0.011 \\
\hline
\end{tabular}


Continuation of Table 3. The coordinates of sixteen wall markers and eleven characteristic points with measurement tolerances $\Delta \mathrm{X}, \Delta \mathrm{Y}, \Delta \mathrm{S}, \Delta \mathrm{Z}$.

\begin{tabular}{|c|c|c|c|c|c|c|c|c|c|}
\hline \multicolumn{10}{|c|}{ No. 2} \\
\hline $\mathrm{X}, \mathrm{m}$ & $\mathrm{Y}, \mathrm{m}$ & $\mathrm{Z}, \mathrm{m}$ & $\mathrm{X}, \mathrm{m}$ & $\mathrm{Y}, \mathrm{m}$ & $\mathrm{Z}, \mathrm{m}$ & $\Delta \mathrm{X}, \mathrm{m}$ & $\Delta \mathrm{Y}, \mathrm{m}$ & $\Delta \mathrm{S}, \mathrm{m}$ & $\Delta \mathrm{Z}, \mathrm{m}$ \\
\hline 99.0440 & 89.7330 & 104.5030 & 99.0452 & 89.7403 & 104.5640 & -0.001 & -0.007 & 0.007 & -0.061 \\
\hline \multicolumn{10}{|c|}{ No. 3} \\
\hline $\mathrm{X}, \mathrm{m}$ & $\mathrm{Y}, \mathrm{m}$ & $\mathrm{Z}, \mathrm{m}$ & $\mathrm{X}, \mathrm{m}$ & $\mathrm{Y}, \mathrm{m}$ & $\mathrm{Z}, \mathrm{m}$ & $\Delta \mathrm{X}, \mathrm{m}$ & $\Delta \mathrm{Y}, \mathrm{m}$ & $\Delta \mathrm{S}, \mathrm{m}$ & $\Delta \mathrm{Z}, \mathrm{m}$ \\
\hline 99.1260 & 89.5480 & 101.3370 & 99.1280 & 89.5520 & 101.3280 & -0.002 & -0.004 & 0.004 & 0.009 \\
\hline \multicolumn{10}{|c|}{ No. 4} \\
\hline $\mathrm{X}, \mathrm{m}$ & $\mathrm{Y}, \mathrm{m}$ & $\mathrm{Z}, \mathrm{m}$ & $\mathrm{X}, \mathrm{m}$ & $\mathrm{Y}, \mathrm{m}$ & $\mathrm{Z}, \mathrm{m}$ & $\Delta \mathrm{X}, \mathrm{m}$ & $\Delta \mathrm{Y}, \mathrm{m}$ & $\Delta \mathrm{S}, \mathrm{m}$ & $\Delta \mathrm{Z}, \mathrm{m}$ \\
\hline 100.3340 & 90.1980 & 104.4400 & 100.3280 & 90.1909 & 104.4740 & 0.006 & 0.007 & 0.009 & -0.034 \\
\hline \multicolumn{10}{|c|}{ No. 5} \\
\hline $\mathrm{X}, \mathrm{m}$ & $\mathrm{Y}, \mathrm{m}$ & $\mathrm{Z}, \mathrm{m}$ & $\mathrm{X}, \mathrm{m}$ & $\mathrm{Y}, \mathrm{m}$ & $\mathrm{Z}, \mathrm{m}$ & $\Delta \mathrm{X}, \mathrm{m}$ & $\Delta \mathrm{Y}, \mathrm{m}$ & $\Delta \mathrm{S}, \mathrm{m}$ & $\Delta \mathrm{Z}, \mathrm{m}$ \\
\hline 99.2970 & 91.0170 & 104.6890 & 99.3001 & 91.0049 & 104.7070 & -0.003 & 0.012 & 0.012 & -0.018 \\
\hline \multicolumn{10}{|c|}{ No. 6} \\
\hline $\mathrm{X}, \mathrm{m}$ & $\mathrm{Y}, \mathrm{m}$ & $\mathrm{Z}, \mathrm{m}$ & $\mathrm{X}, \mathrm{m}$ & $\mathrm{Y}, \mathrm{m}$ & $\mathrm{Z}, \mathrm{m}$ & $\Delta \mathrm{X}, \mathrm{m}$ & $\mathrm{m}$ & $\Delta \mathrm{S}, \mathrm{m}$ & $\Delta \mathrm{Z}, \mathrm{m}$ \\
\hline 101.4630 & 94.7160 & 104.5500 & 101.4680 & 94.7023 & 104.5910 & -0.005 & 0.014 & 0.015 & -0.041 \\
\hline \multicolumn{10}{|c|}{ No. 7} \\
\hline $\mathrm{X}, \mathrm{m}$ & $\mathrm{Y}, \mathrm{m}$ & $\mathrm{Z}, \mathrm{m}$ & $\mathrm{X}, \mathrm{m}$ & $\mathrm{Y}, \mathrm{m}$ & $\mathrm{Z}, \mathrm{m}$ & $\Delta \mathrm{X}, \mathrm{m}$ & $\Delta \mathrm{Y}, \mathrm{m}$ & $\Delta \mathrm{S}, \mathrm{m}$ & $\Delta \mathrm{Z}, \mathrm{m}$ \\
\hline 105.5350 & 100.5440 & 107.2060 & 105.5150 & 100.5670 & 107.2190 & 0.020 & -0.023 & 0.030 & -0.013 \\
\hline \multicolumn{10}{|c|}{ No. 8} \\
\hline $\mathrm{X}, \mathrm{m}$ & $\mathrm{Y}, \mathrm{m}$ & $Z, m$ & $\mathrm{X}, \mathrm{m}$ & $\mathrm{Y}, \mathrm{m}$ & $\mathrm{Z}, \mathrm{m}$ & $\Delta \mathrm{X}, \mathrm{m}$ & $\Delta \mathrm{Y}, \mathrm{m}$ & $\Delta \mathrm{S}, \mathrm{m}$ & $\Delta \mathrm{Z}, \mathrm{m}$ \\
\hline 108.4370 & 110.0840 & 104.7800 & 108.4380 & 110.0840 & 104.8120 & -0.001 & 0.000 & 0.001 & -0.032 \\
\hline \multicolumn{10}{|c|}{ No. 9} \\
\hline $\mathrm{X}, \mathrm{m}$ & $\mathrm{Y}, \mathrm{m}$ & $\mathrm{Z}, \mathrm{m}$ & $\mathrm{X}, \mathrm{m}$ & $\mathrm{Y}, \mathrm{m}$ & $Z, m$ & $\Delta \mathrm{X}, \mathrm{m}$ & $\Delta \mathrm{Y}, \mathrm{m}$ & $\Delta \mathrm{S}, \mathrm{m}$ & $\Delta \mathrm{Z}, \mathrm{m}$ \\
\hline 114.8790 & 97.4580 & 104.3710 & 114.8850 & 97.4851 & 104.4900 & -0.006 & -0.027 & 0.028 & -0.119 \\
\hline \multicolumn{10}{|c|}{ No. 10} \\
\hline $\mathrm{X}, \mathrm{m}$ & $\mathrm{Y}, \mathrm{m}$ & $Z, m$ & $\mathrm{X}, \mathrm{m}$ & $\mathrm{Y}, \mathrm{m}$ & $\mathrm{Z}, \mathrm{m}$ & $\Delta \mathrm{X}, \mathrm{m}$ & $\Delta \mathrm{Y}, \mathrm{m}$ & $\Delta \mathrm{S}, \mathrm{m}$ & $\Delta \mathrm{Z}, \mathrm{m}$ \\
\hline 108.0850 & 109.3300 & 99.8010 & 108.0910 & 109.3520 & 99.9256 & -0.006 & -0.022 & 0.023 & -0.125 \\
\hline \multicolumn{10}{|c|}{ No. 11} \\
\hline $\mathrm{X}, \mathrm{m}$ & $\mathrm{Y}, \mathrm{m}$ & $\mathrm{Z}, \mathrm{m}$ & $\mathrm{X}, \mathrm{m}$ & $\mathrm{Y}, \mathrm{m}$ & $\mathrm{Z}, \mathrm{m}$ & $\Delta \mathrm{X}, \mathrm{m}$ & $\Delta \mathrm{Y}, \mathrm{m}$ & $\Delta \mathrm{S}, \mathrm{m}$ & $\Delta \mathrm{Z}, \mathrm{m}$ \\
\hline 101.0570 & 97.9750 & 104.1770 & 101.0600 & 97.9931 & 104.1990 & -0.003 & -0.018 & 0.018 & -0.022 \\
\hline
\end{tabular}

\begin{tabular}{|c|c|c|}
\hline "TOTAL NUMBER OF POINTS (N): & \multicolumn{2}{|c|}{27} \\
\hline$\sum \Delta \mathbf{S}^{2}$ & \multicolumn{2}{|c|}{$0.0048 \mathrm{~m}^{2}$} \\
\hline RMS error $\left(\mathbf{m}_{\Delta \mathrm{S}}\right)$ & \multicolumn{2}{|c|}{$0.01 \mathrm{~m}$} \\
\hline Permissible $\mathbf{m}_{\Delta \mathrm{s}}$ & \multicolumn{2}{|c|}{$0.03 \mathrm{~m}$} \\
\hline$\overline{\sum \Delta \mathbf{Z}}$ & \multicolumn{2}{|c|}{$0.5520 \mathrm{~m}$} \\
\hline Average point error in height $\left(\Theta_{\Delta Z}\right)$ & \multicolumn{2}{|c|}{$0.02 \mathrm{~m}$} \\
\hline \multirow[t]{2}{*}{ Permissible $\Theta_{\Delta z}$} & \multicolumn{2}{|c|}{$0.03 \mathrm{~m}$} \\
\hline & $\Delta \mathbf{S}$ & $\Delta \mathbf{Z}$ \\
\hline Number of points included in the tolerance & $27(100 \%)$ & $27(100 \%)$ \\
\hline
\end{tabular}

The three-dimensional model of a cultural heritage object of regional significance "Outhouse from the Plotnikovs Manor" in the city of Tobolsk was created by order of a construction company for the further development of a reconstruction project. The terms of reference provided for the mean square error of the location of the characteristic points, not exceeding $0.03 \mathrm{~m}$.

The accuracy of this three-dimensional model made it possible to carry out the necessary measurements and draw up a project for further reconstruction of the cultural 
heritage object of regional significance "Outhouse from the Plotnikovs Manor" in the city of Tobolsk (Figure 6).

An analysis of the results obtained by measuring the points showed that the method of obtaining coordinates and heights using the 3D model corresponds to the tolerances established by Russian legislation.

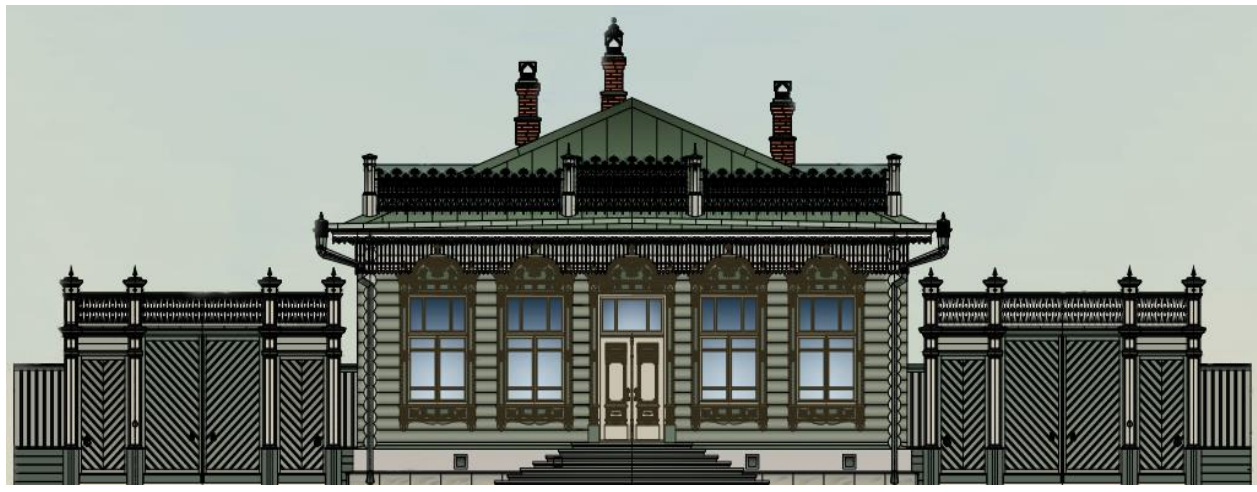

Fig. 6. Graphic part of the reconstruction project.

At present, in Russia much attention is paid to digitalization in the field of geoinformational technologies. The development of modern society led to the fact that the implementation of activities in various fields requires obtaining relevant and reliable information about the state of the world using $3 \mathrm{D}$ models. Thanks to the introduction of uncrewed aerial vehicle, aerial photography become a less time-consuming process. The development of the computer sphere contributed to the emergence of special software that can turn a set of images into a 3D model of a building, structure or the whole city.

Real-time visualization of the $3 \mathrm{D}$ model makes it possible to completely immerse yourself in the model space and thereby transmit the most complete information about the object to the user. This approach allows to gain experience in operating facilities even at the stage of development of their concept. In urban management, the 3D model allows to carry out engineering calculations and to plan social infrastructure [8].

The stereo model allows to determine the horizontal and vertical coordinates of the object at the same time, which eliminates the need to measure the height of the points with geodetic means, using a digital elevation model or horizontally on a graphical plan. The regulatory legal acts contain requirements for the accuracy of determining the coordinates and heights of characteristic points when measuring a stereo model.

For automatic creation of the $3 \mathrm{D}$ model of the cultural heritage object of regional significance "Outhouse from the Plotnikovs Manor" in the city of Tobolsk, the images obtained during planned aerial photography along with perspective (oblique) were used in the ContextCapture program. The obtained three-dimensional model of the object of cultural heritage had high accuracy, the values of $\Delta \mathrm{S}, \Delta \mathrm{Z}$ were included in the tolerance at all selected characteristic points. Also, this $3 \mathrm{D}$ model was used to draw up a project for the reconstruction of the building and allowed to reduce significantly real measurements insitu.

However, to carry out planned aerial photography along with perspective (oblique), it is necessary to fly around the building twice or re-equip the aircraft and then to make a survey with two cameras at once (the axis of one camera is directed vertically downward and the other is tilted). Shooting the facades of each building at different heights and at different angles will take much longer. This type of aerial photography has a higher monetary value compared to the planned one, which is most often used to create stereo models. 
Today, the stereo model is affordable and familiar to the user. It is widely used for complex cadastral works, design, decryption, etc. The requirements for its accuracy are regulated by the legislation in the field of spatial data. At the moment, when automatically creating a 3D model, it is difficult to achieve results close to the stereo model.

The automatic construction of 3D models requires further study, development and improvement through various experiments. In addition, for its wide distribution, it is necessary to begin the development of a special regulatory legal base, as well as provide enterprises with special software products for 3D model creation.

\section{References}

1. Passport of the national project "National Program "Digital Economy of the Russian Federation, Protocol dated 04.06.2019 N 7

2. Federal Law dated December 30, 2015 N 431-FL (as amended on August 3, 2018) "On Geodesy, Cartography, and Spatial Data and on Amending Certain Legislative Acts of the Russian Federation"

3. General Directorate of Geodesy and Cartography under the Council of Ministers of the USSR, 152 (1985)

4. B.N. Akhmedov, Youth Scientific and Practical Conference: Collection of scientific reports, 9 (2017)

5. S.M. Digo, Database. Design and creation: Educational-methodical complex, 171 (2008)

6. A.I. Zhuravlev, Geoprofi: scientific and technical journal on geodesy, cartography and navigation, (2014)

7. M.P. Osipov, O.A. Chekodaev, CEUR Workshop Proceedings, 2212, 359 (2018)

8. M.P. Osipov, O.A. Chekodaev, CEUR Workshop Proceedings, 534 (2019)

9. O.A. Andreeva, S.G. Dyshlenko, ITNOU: Information Technologies in Science, Education and Management, 1, 39 (2019)

10. A.A. Murasheva, P.P. Lepekhin, V.E. Konovalov, A.V. Dontsov, V.V. Vershinin, International Journal of Civil Engineering and Technology, 10 (2), 951 (2019)

11. V.B. Pisetsky, I.A. Savintsev, Yu.V. Patrushev, S.M. Chevdar, Gornyi Zhurnal, 9, 13 (2015) 